\title{
Accuracy of depolarization and delay spread predictions using advanced ray-based modeling in indoor scenarios
}

\author{
Francesco Mani ${ }^{* *}$, François Quitin ${ }^{1,2}$ and Claude Oestges ${ }^{1}$
}

\begin{abstract}
This article investigates the prediction accuracy of an advanced deterministic propagation model in terms of channel depolarization and frequency selectivity for indoor wireless propagation. In addition to specular reflection and diffraction, the developed ray tracing tool considers penetration through dielectric blocks and/or diffuse scattering mechanisms. The sensitivity and prediction accuracy analysis is based on two measurement campaigns carried out in a warehouse and an office building. It is shown that the implementation of diffuse scattering into RT significantly increases the accuracy of the cross-polar discrimination prediction, whereas the delay-spread prediction is only marginally improved.
\end{abstract}

Keywords: Propagation, ray tracing, indoor radio communications, scattering, polarization

\section{Introduction}

The characterization of wireless propagation channels may rely either on stochastic or deterministic models [1]. When site-specific predictions are required, the choice often falls on deterministic models [2], e.g., raybased techniques. In particular, ray tracing $(\mathrm{RT})$ is a geometrical optics approach that evaluates all paths followed by rays as they interact with the environment. It has been successfully used for many years for macro and microcellular channel modeling.

However, wireless systems have continuously evolved, implying that models should evolve too. While a classical deterministic model may have proved accurate for relatively narrowband cellular systems, this might not hold true when dealing with the application-oriented systems exploiting multiple dimensions (space, polarization, frequency, etc.).

When outdoor cellular propagation is modeled by means of 3D RT tools, these usually consider line of sight (LOS) propagation as well as specular reflection and diffraction at various orders, and neglect transmission trough buildings [3]. It has been shown in $[4,5]$

\footnotetext{
* Correspondence: Francesco.Mani@uclouvain.be

'Electrical Engineering Department, ICTEAM Institute, Universitè catholique

de Louvain, Louvain la Neuve, Belgium

Full list of author information is available at the end of the article
}

that such tools are able to accurately predict narrowand wideband propagation characteristics in microcellular outdoor scenarios. However, to correctly reproduce the cross-polar discrimination, improvements are required $[6,7]$ when, for example, transmitter and receiver are at a similar height and most of the reflected and diffracted rays are confined in the horizontal plane.

Indoor path-loss predictions using RT are also usually satisfactory [8-10], at least if penetration is accounted for. This mechanism is particularly important in the absence of LOS or when the terminals are positioned in different rooms. Yet, in situations when the communication takes place along the horizontal plane, it can be expected that the few oblique rays modeled by RT (e.g., the ones reflected by the floor or the ceiling), even when including penetration, might again not be able to predict the polarization behavior [11]. However, only few results can be found on the implementation of diffuse scattering [12] or on polarization characteristics $[13,14]$ in indoor scenarios.

In view of the above discussion, the goal of this study is to compare a classical UTD-based RT tool [15] with an enhanced version incorporating penetration and diffuse scattering models. Two indoor measurement campaigns have been employed to investigate the prediction accuracy on two metrics representative of wideband

\section{SpringerOpen ${ }^{\circ}$}

(c) 2011 Mani et al; licensee Springer. This is an Open Access article distributed under the terms of the Creative Commons Attribution License (http://creativecommons.org/licenses/by/2.0), which permits unrestricted use, distribution, and reproduction in any medium, provided the original work is properly cited. 
dual-polarized systems: delay spread and cross-polarization ratio.

An additional novel aspect of this study is the choice of the scenarios. While the first one is an office building, that is the typical indoor environment investigated when dealing with RT, the second one is a warehouse [16]. To our knowledge, only few articles have considered such industrial environments for RT [17] or for general propagation studies [18]. Nevertheless, such environments become more and more representative of wireless systems deployed to control the storage or industrial processes.

This article is organized as follows. Sections II and III present the penetration and diffuse scattering models, respectively. Section IV describes both the measurement campaigns employed in this study. In Section V, the prediction accuracy of RT simulations with respect to experimental results is investigated. Finally, Section VI summarizes the main conclusions.

\section{Through-block penetration model}

Penetration usually consists of two transmissions through the two sides of a block. In the cases where either the inward or the outward point is a reflection or diffraction point, only one transmission is involved. In order not to affect the image database construction on which the RT tool is based, a simple approximation is made whereby the path direction of a ray that undergoes penetration is not modified by this interaction. The output point and the length of the path are evaluated as if it passes straight through the block. However, the change in the angle of the path of the ray is calculated to evaluate the transmission coefficients.

Further to this, the field at the observation point $P$, after the penetration, can be evaluated as follows [19]:

$$
\mathbf{E}(P)=\mathbf{E}_{0} \cdot \mathbf{T}_{\text {in }} \cdot \mathbf{T}_{\text {out }} \cdot \frac{e^{-j k_{0}\left(d_{1}+d_{3}\right)} e^{-j k_{p} d_{2}}}{d_{1}+d_{2}+d_{3}}
$$

where $\mathbf{E}_{0}$ is the impinging field at the transmitting point, $\mathbf{T}_{\text {in }}$ and $\mathbf{T}_{\text {out }}$ are the dyadic transmission coefficients, $d_{1}$ is the distance between the transmitting point and the incoming penetration point, $d_{2}$ is the distance between the two penetration points, $d_{3}$ is the distance between the outgoing penetration point and the observation point, $k_{0}$ is the propagation constant in free space, and $k_{\mathrm{p}}$ is the propagation constant in the lossy medium, where the penetration occurs.

At each boundary, the dyadic transmission coefficient is decomposed [19] into a perpendicular polarization coefficient:

$$
T_{\perp}=\frac{2 \cos \theta_{i}}{\cos \theta_{i}+\sqrt{\varepsilon_{2 e} / \varepsilon_{1 e}-\sin ^{2} \theta_{i}}}
$$

and a parallel polarization coefficient:

$$
T_{\|}=\frac{2 \sqrt{\varepsilon_{1 e} / \varepsilon_{2 e}} \cos \theta_{i}}{\cos \theta_{i}+\sqrt{\varepsilon_{1 e} / \varepsilon_{2 e}} \sqrt{1-\left(\varepsilon_{1 e} / \varepsilon_{2 e}\right) \sin ^{2} \theta_{i}}}
$$

The effective permittivity for a lossy medium is

$$
\varepsilon_{\mathrm{e}}=\varepsilon_{\mathrm{r}}-j \frac{\sigma}{\omega \varepsilon_{0}}
$$

where $\varepsilon_{0}$ is the vacuum permittivity, and $\varepsilon_{\mathrm{r}}$ and $\sigma$ are the relative permittivity and conductivity of the medium, respectively. The complex propagation constant is $k_{\mathrm{p}}=k_{0} \sqrt{\varepsilon_{\mathrm{e}}}$.

\section{Diffuse scattering model}

The diffuse component of the signal can be defined as the one scattered in directions other than that of the specular one as a result of the interactions with rough surfaces or objects, which cannot be described in the input database of the software. Considering that RT depicts a virtual scenario made by perpendicular parallelepipeds of any orientation, an irregularity can also be an object that diverts from this ideality, e.g., for its shape.

The model implemented into RT tool is based on [20]. In $\mathrm{RT}$, the diffuseness can be created by dividing the surfaces affected by scattering into surface elements or tiles. A diffuse scattering ray is assumed to originate from the center of each surface element. The size of each element is set by the well-known far-field condition [21]:

$$
r>\frac{2 D^{2}}{\lambda}
$$

where $r$ is the distance between the center of the element and the terminal, and $D$ is the dimension of the surface element. Each element is evaluated in the tool by recursively dividing a surface until it meets this farfield condition. Each rectangular surface is divided into four equal rectangle. For each of them, the condition is then checked again. It may be possible that the tiles closer to the virtual transmitter do not meet this condition but the further ones do, leading to a subdivision of a surface into elements of different sizes.

A scattering coefficient $S$ and a scattering pattern model are associated with each surface. The reflection reduction factor, $R$, sets the amount of energy subtracted by the scattering from the specular reflection. The definition of the scattering coefficient implemented in this study is

$$
S=\left.\frac{\left|\mathbf{E}_{s}\right|}{\left|\mathbf{E}_{r}\right|}\right|_{d S}
$$


Following this definition, $R$ can be evaluated as

$$
R=\sqrt{1-S^{2}}
$$

The geometry of the scattering interaction can be seen in [20].

The pattern models allow to estimate the amplitude of the scattered field. As the scattered wave is assumed incoherent, its phase generated randomly with an uniform distribution. In this study, diffuse scattering does not include any model for depolarization. This means that diffuse rays maintain their local polarization.

Two pattern models [20] are implemented in this study:

1) a Lambertian model, with the maximum in the direction perpendicular to the surface;

2) a directive model, with the maximum in the direction of specular reflection

\section{A. Lambertian model}

The radiation lobe of the Lambertian model has its maximum in the direction perpendicular to the surface. The amplitude of the scattered field from a surface element is

$$
d E_{s}^{2}=K_{0}^{2} S^{2} \Gamma^{2} \frac{d S \cos \theta_{i} \cos \theta_{s}}{\pi} \frac{1}{r_{i}^{2} r_{s}^{2}}
$$

where $K_{0}=\sqrt{60 G_{\mathrm{t}} P_{\mathrm{t}}}$ and $G_{\mathrm{t}}$ and $P_{\mathrm{t}}$ are, respectively, the gain and the input power of the transmitting antenna.

\section{B. Directive model}

The scattering lobe is steered toward the direction of the specular reflection. To achieve this, it is possible to write the following expression for the amplitude of the scattered field from a surface element:

$$
d E_{s}^{2}=E_{s 0}^{2} \cdot\left(\frac{1+\cos \psi_{r}}{2}\right)^{\alpha_{r}} \alpha_{r}=1, \ldots, N
$$

where $\psi_{r}$ is the angle between the specular reflection direction and the scattering direction, and $\alpha_{r}$ is a parameter that sets the width of the scattering lobe. The higher the $\alpha_{r}$, the narrower the lobe. The expression of the maximum amplitude $E_{s 0}$ is

$$
E_{s 0}^{2}=\left(\frac{S K}{r_{i} r_{s}}\right)^{2} \Gamma^{2} \frac{d S \cos \theta_{i}}{F_{\alpha_{r}}}
$$

where

$$
F_{\alpha_{r}}=\frac{1}{2^{\alpha_{r}}} \cdot \sum_{j=0}^{\alpha_{r}}\left(\begin{array}{c}
\alpha_{r} \\
j
\end{array}\right) \cdot I_{j}
$$

and $I_{j}$ is then defined as

$$
I_{j}= \begin{cases}\frac{2 \pi}{j+1} & \text { if } j \bmod 2=0 \\ \frac{2 \pi}{j+1} \cdot f\left(\theta_{i}\right) & \text { if } j \bmod 2 \neq 0\end{cases}
$$

where

$$
f\left(\theta_{i}\right)=\cos \theta_{i} \cdot \sum_{\omega=0}^{\frac{j-1}{2}}\left(\begin{array}{c}
2 \omega \\
\omega
\end{array}\right) \cdot \frac{\sin ^{2 \omega} \theta_{i}}{2^{2 \omega}}
$$

\section{Measurement campaigns}

Two indoor measurement campaigns have been used in this study to tune the parameters and carry out the accuracy analysis. The two contrasting scenarios are

- a warehouse scenario,

- an office scenario.

\section{A. Warehouse scenario}

Measurements were carried out at Transport Pierre in Wavre (Belgium) in a warehouse of approximatively 300 $\mathrm{m}^{2}$ with metallic and wooden containers for the storage of furniture. Walls and columns, in the room, are made of concrete. Figure 1 shows the plan of the warehouse and the position of the receiving $(\mathrm{Rx})$ and transmitting (Tx) antennas. The Rx position is fixed, while $\mathrm{Tx}$ is placed in ten different positions, which proceed in a clockwise direction from the position labeled $T x_{1}$ to that of $T x_{10}$. The antennas used for making measurements

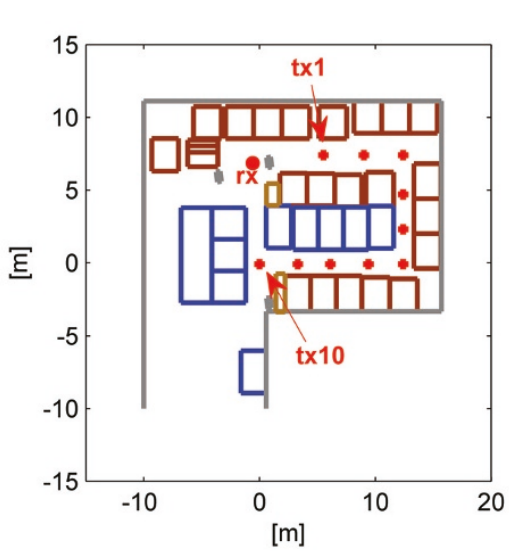

Figure 1 Plan of transport Pierre warehouse where measurements were conducted. 
are linearly polarized, with an azimuth omnidirectional pattern in the frequency range between $800 \mathrm{MHz}$ and 2.5 GHz. The horizontal polarization has been obtained by manual rotation of the antennas. Measurements have been recorded with a SISO Channel Sounder at a frequency of $1.9 \mathrm{GHz}$ with a bandwidth of $80 \mathrm{MHz}$ [22]. The elevation pattern of the antennas at that frequency can be found in [23]. No information on the crosspolar pattern of the antenna is provided by its manufacturer. The measurements of cross-polar discrimination of the antenna have been performed in an anechoic chamber. The mean value is $8.2 \mathrm{~dB}$ with a standard deviation of $6.7 \mathrm{~dB}$ over all angles.

It is noted that the implementation of this scenario must be simplified, mainly for the presence of a great number of objects that could not be modeled into the database, e.g., small boxes on top of the containers.

\section{B. Office scenario}

A. second measurement campaign has been performed in an office environment at the Universitè Libre de Bruxelles (ULB). A floor plan of the measurement environment is given in Figure 2. It consists of a main hall, surrounded by offices. The rooms are filled with tables and furniture, and the walls are composed partly of drywalls and partly of brick walls. The labs and offices are also surrounded by several windows. The door between $\mathrm{Tx}$ and $\mathrm{Rx}$ remained closed for all the measurements in the hall to avoid line-of-sight conditions. At each measurement location, a 2-level virtual uniform planar array (2L-VUPA) was realized using an automatic positioning device, yielding a $10 \times 10 \times 2$ virtual array. The elements of the planar array were spaced by $\lambda / 3$, and the two levels of the $2 \mathrm{~L}-\mathrm{VUPA}$ were spaced by $1.2 \lambda$. The $\mathrm{Rx}$ antenna was a tri-pole antenna, composed of three perpendicularly polarized short linear antennas. The $\theta$-and

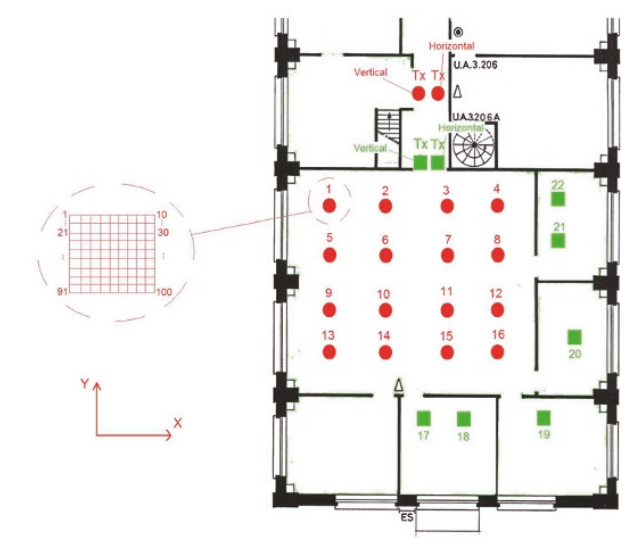

Figure 2 Floor plan of the office environment. $\varphi$-radiation patterns of the three antennas of the tri-pole have been measured in an anechoic chamber [24], and the 3D plots are shown in [25]. The Tx antennas consisted of two log-periodic antennas, one vertically polarized and one horizontally polarized, that were spaced a few wavelengths apart. The crosspolar discrimination of these antennas is larger than $15 \mathrm{~dB}$.

At each position of the 2L-VUPA, the frequency response was measured simultaneously on the three $\mathrm{Rx}$ antennas with a 4-port Rhode\&Schwarz ZVA-24 vector network analyzer (VNA), while switching between the two Tx antennas using a fast switching device. The central working frequency of the VNA was $3.6 \mathrm{GHz}$, with a bandwidth of $200 \mathrm{MHz}$. The measurements were performed at night, so that the environment remained entirely static between measurements.

\section{RT Results}

Results are presented in terms of delay spread, $t_{\mathrm{ds}}$, and cross-polarization ratio (XPR), denoted as $\chi$. First, $\chi$ may be defined as

$$
\chi=10 \log \left(\frac{P_{x p}}{P_{\mathrm{CO}}}\right)
$$

where $P_{\mathrm{xp}}$ is the cross-polar power at the $\mathrm{Rx}$, and $P_{\text {co }}$ is the copolar power at the Rx. The delay spread may then be defined as [26]

$$
t_{\mathrm{ds}}=\sqrt{\frac{\sum_{i}\left(t_{i}-\frac{\sum_{i} t_{i} P_{i}}{\sum_{i} P_{i}}\right)^{2} P_{i}}{\sum_{i} P_{i}}}
$$

where $t_{i}$ is the time of arrival of the $i$ th ray, and $P_{i}$ is the power related to that ray.

The simulated 3D environment is created taking into account constituent parts of buildings (i.e., walls, ceiling, and floor) and major objects present in the scenario (i. e., the containers in warehouse scenario and doors and desks in the office scenario). Unless otherwise stated, a maximum of three reflections and a single diffraction are utilized. Diffuse scattering is also included by considering a maximum of two interactions in the simulations. It means that single scattering and scattering before or after a single reflection are included. Penetration is also included unless otherwise stated.

Each delay spread is an average over five values evaluated in an area with a radius of $15 \mathrm{~cm}$ around the nominal position, to overcome fading due to error in the positioning. Each XPR calculation, instead, is an average over $15 \mathrm{XPR}$ values in the same position to overcome the arbitrariness introduced by scattering random phase.

In both scenarios, the evaluations begin with a sensitivity test for delay spread and XPR with differing values 
of the diffuse scattering parameter $S$ and for different scattering pattern models. The model is then validated through comparisons with measurements.

\section{A. Warehouse scenario}

The nominal permittivity, $\varepsilon_{\mathrm{r}}$, and conductivity, $\sigma$, values of the materials present in the warehouse are $[27] \varepsilon_{\mathrm{r}}=9$ and $\sigma=9 \cdot 10^{-2}[\mathrm{~S} / \mathrm{m}]$ for concrete walls, respectively, and $\varepsilon_{\mathrm{r}}=2$ and $\sigma=2 \cdot 10^{-3}[\mathrm{~S} / \mathrm{m}]$ for wooden containers, respectively.

Table 1 shows mean errors in XPR prediction for each value of the scattering coefficient and for each pattern model after the sensitivity test. Three directive models, distinguished by $\alpha_{\mathrm{r}}$ values, are tested. The row entitled "Pattern Error" refers to the average error for each pattern model over the different values of $S$. The column entitled "S Error" is the analog for the scattering coefficients.

A similar comparison has been made for delay spread results. Table 2 shows the relative mean errors for delay spread prediction, with respect to the measured delay spread value in each location.

Some conclusions can be drawn from these tests:

- The Lambertian model gives the best results in predicting both the XPR and the delay spread in this environment.

- For XPR, the improvement with a Lambertian model is significant, while for delay spread, it is less important.

- Scattering coefficient variations do not greatly influence the prediction errors.

- Considering Lambertian model, the higher the $S$, the lower the errors.

For the following results, a Lambertian pattern model with $S=0.4$, which gives an error of $3.3 \mathrm{~dB}$ with a standard deviation of 2.3 for XPR prediction and an absolute error of 6.9 ns with a standard deviation of 4.4 for delay spread prediction, has been chosen. The choice of a Lambertian model, where the amplitude of the scattered ray does not depend on the direction of the impinging wave, can be also explained by considering the nature of the scenario where there are a great number of smaller

Table 1 Cross-polarization mean error in warehouse scenario (dB)

\begin{tabular}{cccccc}
\hline & Lambertian & $\boldsymbol{\alpha}_{\mathbf{r}}=\mathbf{2}$ & $\boldsymbol{\alpha}_{\mathrm{r}}=\mathbf{3}$ & $\boldsymbol{\alpha}_{\mathrm{r}}=\mathbf{4}$ & $\boldsymbol{S}$ Error \\
\hline$S=0.2$ & 5.1 & 4.9 & 4.1 & 5.8 & 5 \\
\hline$S=0.3$ & 4.3 & 5.3 & 6.9 & 5.3 & 5.4 \\
\hline$S=0.4$ & 3.3 & 5.6 & 7.6 & 4.8 & 5.3 \\
\hline Pattern error & 4.2 & 5.3 & 6.2 & 5.3 & \\
\hline
\end{tabular}

Table 2 Delay spread relative mean error in warehouse scenario

\begin{tabular}{cccccc}
\hline & Lambertian & $\boldsymbol{\alpha}_{\mathrm{r}}=\mathbf{2}$ & $\boldsymbol{\alpha}_{\mathrm{r}}=\mathbf{3}$ & $\boldsymbol{\alpha}_{\mathrm{r}}=\mathbf{4}$ & $\boldsymbol{S}$ Error \\
\hline$S=0.2$ & 0.36 & 0.35 & 0.36 & 0.37 & 0.36 \\
\hline$S=0.3$ & 0.33 & 0.35 & 0.36 & 0.36 & 0.35 \\
\hline$S=0.4$ & 0.33 & 0.34 & 0.35 & 0.36 & 0.345 \\
\hline Pattern error & 0.34 & 0.347 & 0.347 & 0.363 & \\
\hline
\end{tabular}

objects that could not be modeled. This may be the reason for the Lambertian behavior of the scattering rays.

Figure 3 shows the comparison between measurements and simulations for XPR. The mean prediction error after the scattering implementation is $3.3 \mathrm{~dB}$ as shown in Table 1 . The importance of including diffuse scattering to accurately predict XPR in this scenario is clear, as otherwise, the software would not be able to model the power transferred between the two polarizations.

Figure 4 shows the comparison between measurements and simulations for delay spread. There appears to be an improvement in the prediction error, mainly in deep non-LOS (NLOS) positions (between 5 and 8). Nevertheless, the measurements are generally underestimated by simulations.

If penetration is not included in the software, in this particular scenario, then the prediction accuracy does not suffer any degradation. In the case of the delay spread, the prediction error does not increase, and in the case of the XPR, the prediction error increases by only $0.3 \mathrm{~dB}$. It can be concluded that penetration is not significant if diffuse scattering is included in the software. This can be explained by considering the row of metallic containers that separates the two terminals when they are in opposite corridors of the warehouse, i. e., the scenario where penetration could have been significant.

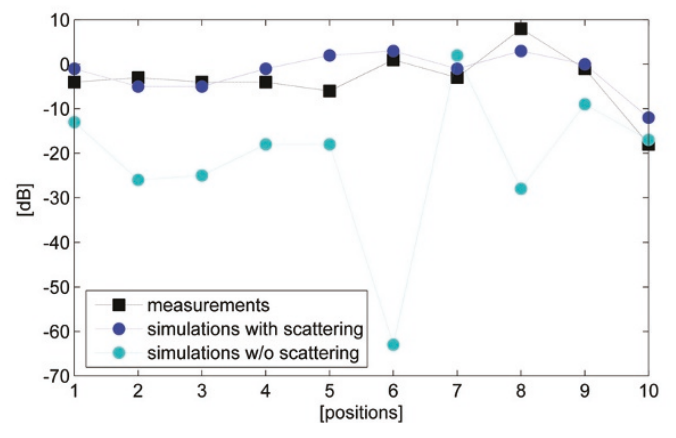

Figure 3 Comparison of XPR simulation results with measurements in warehouse scenario. 


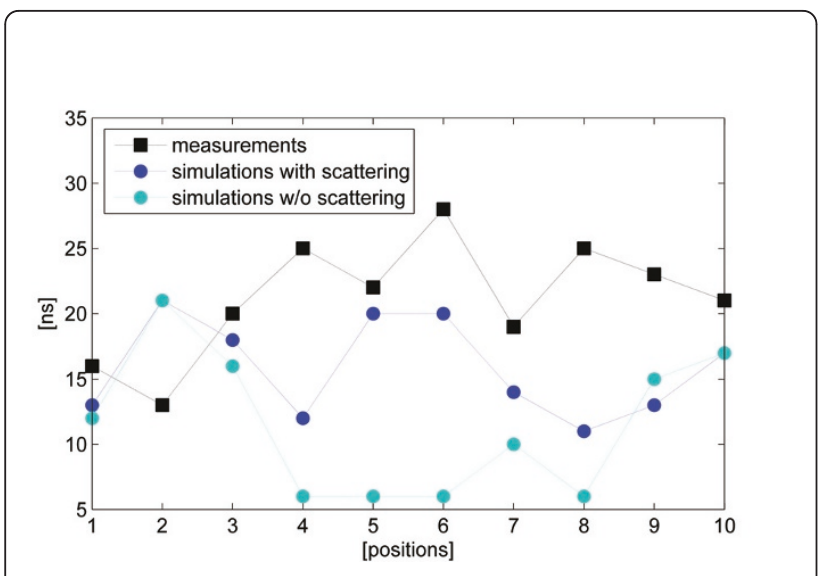

Figure 4 Comparison of delay spread simulation results with measurements in warehouse scenario.

To verify a possible improvement in the delay spread prediction, the maximum number of reflections has been increased from three to four. The outcome is that there is no significant improvement in the prediction. Moreover, the addiction of one more interaction to the maximum number of reflections incurs impractical computational times.

\section{B. Office scenario}

It is important to note that without the implementation of penetration, in this office scenario, no results could be obtained in any position since the $\mathrm{Rx}$ and $\mathrm{Tx}$ are always separated by a door or a wall. Table 3 shows the permittivity, $\varepsilon_{\mathrm{r}}$, and conductivity, $\sigma$, the values of the materials present in the propagation scenario at 3.6 GHz.

Unless otherwise stated, in the XPR definition of (14), $P_{\mathrm{xp}}$ is the power collected by the Rx antenna along $\vec{x}$, considering the coordinate system shown in Figure 2. In this study, the Tx antenna is always the vertically polarized one.

By analogy with the warehouse scenario, Table 4 shows the variations of XPR prediction mean error with the scattering parameters. The columns entitled $\alpha_{\mathrm{r}}$ refer to a directive pattern model with the relative lobe width.

Table 3 Materials' properties at $3.6 \mathrm{GH}_{\mathrm{Z}}[\mathbf{2 9 - 3 2}]$

\begin{tabular}{ccc}
\hline & $\boldsymbol{\varepsilon}_{\mathbf{r}}$ & $\boldsymbol{\sigma}[\mathrm{S} / \mathrm{m}]$ \\
\hline Brick & 4.5 & $1 \cdot 10^{-2}$ \\
\hline Concrete walls & 7 & $4 \cdot 10^{-3}$ \\
\hline Drywall & 2.3 & $1 \cdot 10^{-5}$ \\
\hline Glass & 4 & - \\
\hline Wood & 2 & $2 \cdot 10^{-3}$ \\
\hline
\end{tabular}

Table 4 Cross-polarization mean error in office scenario (dB)

\begin{tabular}{cccccc}
\hline & Lambertian & $\boldsymbol{\alpha}_{\mathrm{r}}=\mathbf{2}$ & $\boldsymbol{\alpha}_{\mathrm{r}}=\mathbf{3}$ & $\boldsymbol{\alpha}_{\mathrm{r}}=\mathbf{4}$ & $\boldsymbol{S}$ Error \\
\hline$S=0.2$ & 6.5 & 6.3 & 5.7 & 5.8 & 6.1 \\
\hline$S=0.3$ & 5.7 & 6.2 & 5.4 & 5.7 & 5.5 \\
\hline$S=0.4$ & 6.6 & 5 & 6.6 & 4.8 & 5.7 \\
\hline Pattern error & 6.3 & 5.8 & 5.9 & 5.4 & \\
\hline
\end{tabular}

Table 5 shows relative mean errors in the delay spread prediction for each value of the scattering coefficient and for each pattern model.

Looking at Table 5, it is clear that in this scenario, the choice between different scattering coefficients and models does not influence significantly the prediction error for delay spread. This error, in any circumstance, is about $30 \%$. Considering XPR prediction, it is possible to conclude that the choice of $S$ does not change the error significantly, but on the other hand, directive models with an higher value of $\alpha_{\mathrm{r}}$ seem to perform better. This means that the diffuse rays with higher power are located close to the direction of specular reflection. A similar conclusion has been drawn in [28] utilizing parameter extraction from the measurements performed in the same scenario, where strongest diffuse power components were found to be spatially co-located with specular channel components, which justifies the use of the directive model.

Simulated results with and without the implementation of diffuse scattering are now presented to validate the model. If scattering is not included, then no ray is predicted arriving at the $\mathrm{Rx}$ in positions 20 and 21 . According to the results of the sensitivity test on scattering parameters, the chosen model is the Directive model with $\alpha_{\mathrm{r}}=4$ and $S=0.4$, which gives an error of $4.8 \mathrm{~dB}$ with a standard deviation of 3.7 for XPR prediction and an absolute error of $5.8 \mathrm{~ns}$ with a standard deviation of 4.7 for delay spread prediction. The choice of this model can also be intuitively justified by the few objects that could not be modeled and by the small irregularities in the surfaces.

Figure 5 shows the comparison between measurements and simulations for $S$. The results show a general improvement when scattering is included in the simulations. In particular, the improvement is significant in

Table 5 Delay spread relative mean error in office scenario

\begin{tabular}{cccccc}
\hline & Lambertian & $\boldsymbol{\alpha}_{\mathrm{r}}=\mathbf{2}$ & $\boldsymbol{\alpha}_{\mathrm{r}}=\mathbf{3}$ & $\boldsymbol{\alpha}_{\mathrm{r}}=\mathbf{4}$ & $\boldsymbol{S}$ Error \\
\hline$S=0.2$ & 0.29 & 0.3 & 0.29 & 0.3 & 0.295 \\
\hline$S=0.3$ & 0.29 & 0.3 & 0.3 & 0.32 & 0.303 \\
\hline$S=0.4$ & 0.3 & 0.31 & 0.29 & 0.3 & 0.3 \\
\hline Pattern error & 0.293 & 0.303 & 0.293 & 0.307 & \\
\hline
\end{tabular}




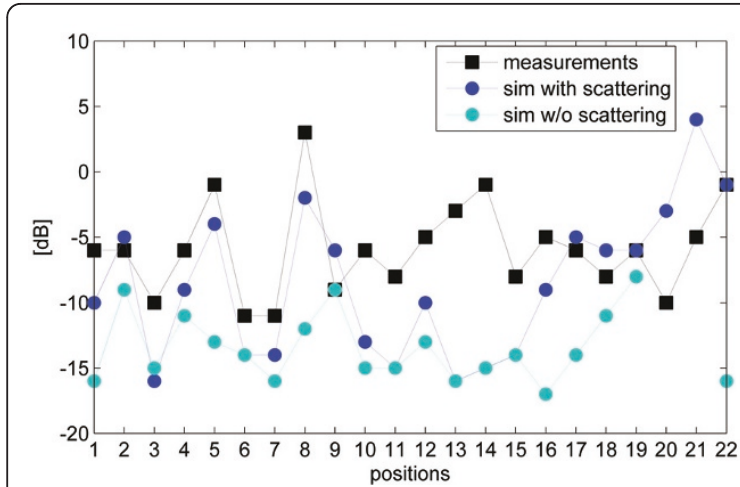

Figure 5 Comparison of XPR simulations results with measurements in office scenario.

the first two rows of measurements in the hall (positions 1-8) and in the offices (positions 17-22). On the other hand, in the last two rows of measurements (positions 9- 16) in the hall, the accuracy is not improved significantly when diffuse scattering is included. The mean prediction error is $4.8 \mathrm{~dB}$ as shown in Table 4 .

Figure 6 shows the comparison between measurements and simulations for the delay spread. The results show that including scattering does not improve significantly the prediction accuracy, except in the last three positions, which can described as deep NLOS, where RT without scattering fails to predict any ray, or a significant number of rays, arriving at the Rx.

Figure 7 shows the XPR comparison where $P_{\mathrm{xp}}$ refers to the power collected by the $\mathrm{Rx}$ antenna along $\vec{\gamma}$, rather than $\vec{x}$ as had been the case in the previous results. Similar conclusions to the ones presented for the other polarization of $\mathrm{Rx}$ antenna may be drawn. The difference is that the mean prediction error, when scattering is included, drops to $3.3 \mathrm{~dB}$, and in this case, the software without scattering fails also in some NLOS positions in the main hall.

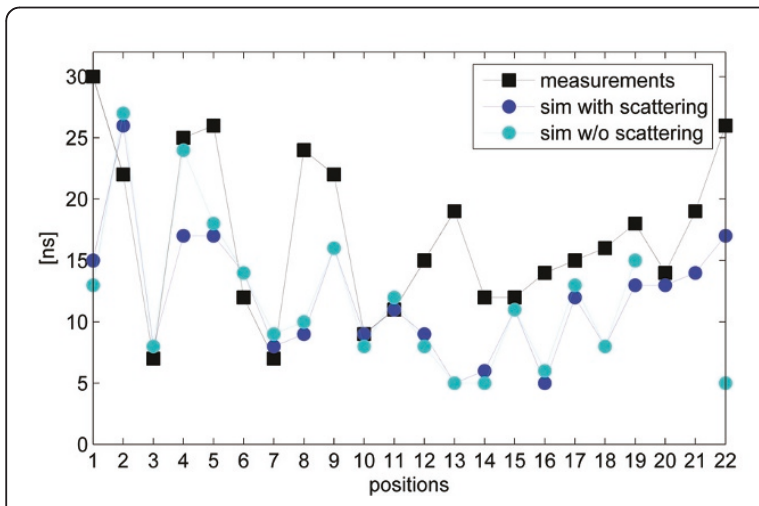

Figure 6 Comparison of delay spread simulations results with measurements in office scenario.

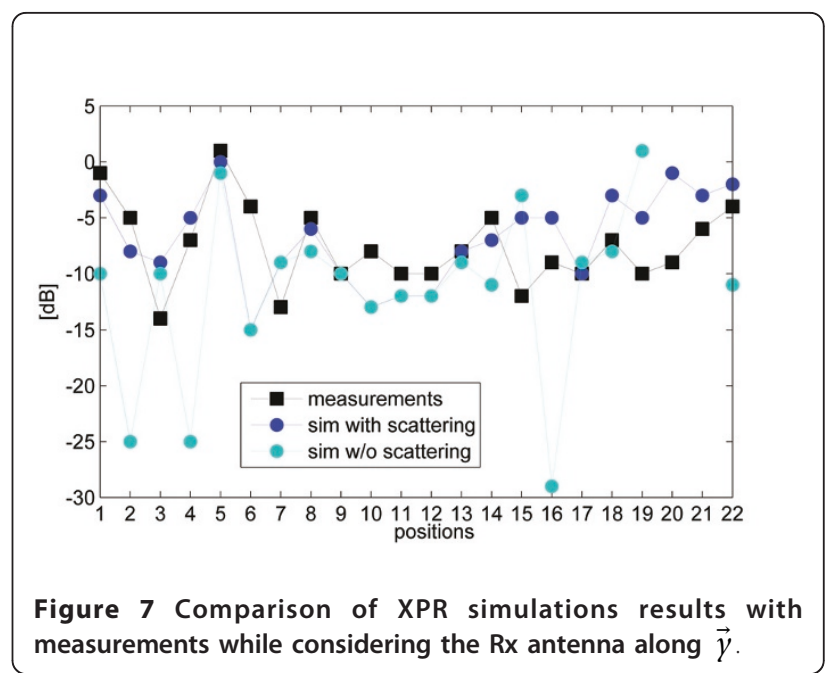

As in the warehouse scenario, to verify a possible improvement in the delay spread prediction, the maximum number of reflections has been increased from three to four. This results in an improvement, but the latter is minimal and present only in few locations. The relative mean error decreases by $2 \%$, whereas the computation time increases by $300 \%$.

\section{Conclusion}

This study has investigated the prediction accuracy of UTD-based RT including penetration and diffuse scattering models in the framework of dual-polarized broadband systems. To this end, two indoor measurements campaigns have been analyzed. The first one was conducted in a warehouse used for the storage of small containers, and the second one in the offices of a university building. Based on the obtained results, it can be concluded that penetration is not relevant in the warehouse scenario because of the geometry of the room and the material of the containers, as a row of metallic containers separates the two terminals when they are in opposite parts of the warehouse, thus preventing any penetration. On the other hand, penetration is essential in the office scenario where $\mathrm{Tx}$ and $\mathrm{Rx}$ are always located in different rooms, thus being separated by a door or a wall.

For both scenarios, a sensitivity test on the diffuse scattering coefficient and pattern model has been performed. While the prediction error does not depend significantly on $S$, the results of the test on pattern models are interesting. In the warehouse scenario, the Lambertian model gives the closest prediction to the measurements. By contrast, in the office scenario, the best result is achieved by a Directive model with a high value of $\alpha_{\mathrm{r}}$, i.e., a narrow beam diffusion. This difference is intuitive. In the warehouse scenario, a lot of large objects could 
not be modeled in the input database, and some surfaces, such as the ceiling, have strong irregularities. In the office, the irregularities are fewer and smaller. It is consistent, then, that the diffuse rays with higher energy are closer to the direction of specular reflection in this case, while they are more randomly distributed in the warehouse.

Comparing the simulation results with measurements, the following conclusions, valid for both scenarios, may be drawn:

- The implementation of diffuse scattering is significant for modeling polarization behavior of the channel (e.g. the power transferred from one polarization to another) with a high degree of accuracy.

- The implementation of diffuse scattering is important in the delay spread prediction only in deep NLOS positions, where diffuse components can be of the same order of magnitude of the coherent ones.

- In LOS (or obstructed LOS in the office scenario) positions, the classic RT tool (neglecting diffuse scattering) is as accurate as the advanced version.

\section{Abbreviations}

2L-VUPA: 2-level virtual uniform planar array; LOS: line of sight; RT: ray tracing; VNA: vector network analyzer; XPR: cross-polarization ratio.

\section{Acknowledgements}

The authors would like to thank Olivier Renaudin and David Gonzalez Ovejero (ICTEAM- Electrical Engineering, UCLouvain) for their help in conducting the warehouse measurement campaign, and Vittorio Degli Esposti and Enrico Maria Vitucci (DEIS, University of Bologna) for their significant input into the implementation of diffuse scattering model. This study was partially supported by the Région Wallone in the framework of WIST-2 project BIRADAR (contract no. 616417) and by the European Commission in the framework of FP7 BuNGee project (contract no. 248267). Claude Oestges and François Quitin are also pleased to acknowledge the financial support of the Belgian Fonds de la Recherche Scientifique.

\section{Author details}

${ }^{1}$ Electrical Engineering Department, ICTEAM Institute, Universitè catholique de Louvain, Louvain la Neuve, Belgium ${ }^{2}$ OPERA Department, Universitè Libre de Bruxelles, Brussels, Belgium

\section{Competing interests}

The authors declare that they have no competing interests.

Received: 7 December 2010 Accepted: 14 June 2011

Published: 14 June 2011

\section{References}

1. M Iskander, Z Yun, Propagation prediction models for wireless communication systems. IEEE Trans Microw Theory Tech. 50(3), 662-673 (2002)

2. S Seidel, T Rappaport, Site-specific propagation prediction for wireless inbuilding personal communication system design. IEEE Trans Vehic Technol. 43(4), 879-891 (1994)

3. L Barclay, Propagation of Radiowaves, (The Institution of Electrical Engineers 2003)
4. G Athanasiadou, A Nix, J McGeehan, A microcellular ray-tracing propagation model and evaluation of its narrow-band and wide-band predictions. IEEE J Sel Areas Commun. 18(3), 322-335 (2000)

5. G Athanasiadou, A Nix, Investigation into the sensitivity of the power predictions of a microcellular ray tracing propagation model. IEEE Trans Vehic Technol. 49(4), 1140-1151 (2000)

6. V Degliesposti, V Kolmonen, E Vitucci, F Fuschini, P Vainikainen, Analysis and ray tracing modelling of co- and cross-polarization propagation in urban environment, in 2nd European Conference on Antennas and PropagationEUCAP, Edinburgh, Scotland, November 2007

7. I Cuinas, M Sanchez, A Alejos, Depolarization due to scattering on walls in the $5 \mathrm{GHz}$ band. IEEE Trans Antenn Propag. 57(6), 1804-1812 (2009)

8. AO Kaya, L Greenstein, W Trappe, Characterizing indoor wireless channels via ray tracing, and validation via measurements, in IEEE Global Telecommunications Conference, New Orleans, USA, December 2008

9. T Rautiainen, $\mathrm{R}$ Hoppe, $\mathrm{G}$ Wölfle, Measurements and $3 \mathrm{D}$ ray tracing propagation predictions of channel characteristics in indoor environments, in IEEE Symposium on Personal, Indoor and Mobile Radio Communications, Athens, Greece, 3-7, September 2007

10. F Saez de Adana, O Gutierrez Blanco, I Gonzalez Diego, J Perez Arriaga, M Catedra, Propagation model based on ray tracing for the design of personal communication systems in indoor environments. IEEE Trans Vehic Technol. 49(6), 2105-2112 (2000)

11. D Chizhik, J Ling, RA Valenzuela, The effect of electric field polarization on indoor propagation, in IEEE International Conference on Universal Personal Communications, Florence, Italy, 5-9, October 1998

12. Y Lostanlen, G Gougeon, Introduction of diffuse scattering to enhance raytracing methods for the analysis of deterministic indoor UWB radio channels, in International Conference on Electromagnetics in Advanced Applications, ICEAA, 903-906, September 2007

13. S Loredo, L Valle, I Paez, R Torres, Accuracy analysis of GO/UTD radio channel modelling in indoor scenarios including cross-polarisation, in IEEE VTS 53rd Vehicular Technology Conference. VTC 2001 Spring. 1, 469-473 (2001)

14. G Athanasiadou, A Nix, A novel 3-D indoor ray-tracing propagation model: the path generator and evaluation of narrow-band and wide-band predictions. IEEE Trans Vehic Technol. 49(4), 1152-1168 (2000)

15. C Oestges, B Clerckx, L Raynaud, D Vanhoenacker-Janvier, Deterministic channel modeling and performance simulation of microcellular wide-band communication systems. IEEE Trans Vehic Technol. 51(6), 1422-1430 (2002)

16. F Mani, C Oestges, Evaluation of diffuse scattering contribution for delay spread and crosspolarization ratio prediction in an indoor scenario, in 4th European Conference on Antennas and Propagation -EUCAP, Barcelona, Spain, 12-16, April 2010

17. P Bosselmann, Planning and analysis of UHF RFID systems for consumer goods logistics using ray tracing predictions, in 1st Annual RFID Eurasia Conference, 1-7, September 2007

18. E Tanghe, J Wout, L Martens, H Capoen, K Van Herwegen, W Vantomme, Large-scale fading in industrial environments at wireless communication frequencies, in Antennas and Propagation Society International Symposium, Honolulu, Hawaii, USA, June 2007

19. U Inan, A Inan, Electromagnetic Waves (Prentice Hall)

20. V DegliEsposti, F Fuschini, EM Vitucci, G Falciasecca, Measurement and modelling of scattering from buildings. IEEE Trans Antenn Propag. 55(1), 143-154 (2007)

21. V DegliEsposti, F Fuschini, EM Vitucci, G Falciasecca, Speed-up techniques for ray tracing field prediction models. IEEE Trans Antenn Propag. 57(5), 1469-1480 (2009)

22. S Haese, C Moullec, P Coston, K Sayegrih, High-resolution spread spectrum channel sounder for wireless communications systems, in IEEE International Conference on Personal Wireless Communication, 170-173 (1999)

23. SMT-8TO25M-A SkyCross antenna, http://www.skycross.com/Products/PDFs/ SMT-8TO25M-A.pdf

24. F Gallee, Development of an isotropic sensor 2-6 GHz, in European Microwave Conference, 91-93 (2007)

25. F Quitin, C Oestges, F Horlin, P De Doncker, Clustered channel characterization for indoor polarized mimo systems, in IEEE 20th International Symposium on Personal, Indoor and Mobile Radio Communications, 1064-1068, September 2009

26. SR Saunders, Antennas and Propagation for Wireless Communication Systems (Wiley 1999) 
27. F Mani, C Oestges, A ray based indoor propagation model including depolarizing penetration, in 3rd European Conference on Antennas and Propagation-EuCAP, Berlin, Germany, March 2009

28. F Quitin, C Oestges, F Horlin, P De Doncker, A spatio-temporal channel model for modeling the diffuse multipath component in indoor environments, in 4th European Conference on Antennas and PropagationEuCAP, Barcelona, Spain, 12-16, April 2010

29. R Wilson, Propagation losses through common building materials. http:// www.skycross.com/Products/PDFs/SMT-8TO25M-A.pdf

30. O Landron, M Feuerstein, T Rappaport, A comparison of theoretical and empirical reflection coefficients for typical exterior wall surfaces in a mobile radio environment. IEEE Trans Antenn Propag. 44(3), 341-351 (1996)

31. H Bertoni, Radio Propagation for Modern Wireless Systems (Prentice Hall 2000)

32. J Baker Jarvis, M Janeziw, B Riddle, R Johnk, P Kabos, C Holloway, R Gever, C Grosvenor, Measuring the permittivity and permeability of lossy materials: solids, liquids, metals, building materials, and negative-index materials. National Institute of Standards and Technology, USA, Technical Report, 1536, February 2005

doi:10.1186/1687-1499-2011-11

Cite this article as: Mani et al: Accuracy of depolarization and delay spread predictions using advanced ray-based modeling in indoor scenarios. EURASIP Journal on Wireless Communications and Networking 2011 2011:11.

\section{Submit your manuscript to a SpringerOpen ${ }^{\mathcal{O}}$ journal and benefit from:}

- Convenient online submission

- Rigorous peer review

- Immediate publication on acceptance

- Open access: articles freely available online

- High visibility within the field

- Retaining the copyright to your article

Submit your next manuscript at $\gg$ springeropen.com 